\title{
Non-invasive oral cancer detection from saliva using zinc oxide-reduced graphene oxide nanocomposite based bioelectrode
}

\author{
Shilpi Verma and Surinder P. Singh, Academy of Scientific and Innovative Research (AcSIR), CSIR-HRDC Campus, Ghaziabad, Uttar Pradesh-201002, \\ India; CSIR-National Physical Laboratory (Campus), Dr. K. S. Krishnan Road, New Delhi 110012, India \\ Address all correspondence to Surinder P. Singh at singh.uprm@gmail.com
}

(Received 21 June 2019; accepted 1 October 2019)

\begin{abstract}
Multifunctional materials with excellent biocompatibility and electron-transport properties are critical for the pursuit of point-of-care biosensing devices. The authors report the synthesis of zinc oxide-reduced graphene oxide (ZnO-rGO) nanocomposite for the fabrication of an electrochemical immunosensing test-bed for noninvasive onsite detection of oral cancer biomarker (interleukin-8, IL8). The immunosensor showed successful detection of IL8 at low concentration ranges, i.e., $100 \mathrm{fg} / \mathrm{mL}-5 \mathrm{ng} / \mathrm{mL}$ with a sensitivity of $12.46 \pm 0.82 \mu \mathrm{ALL} / \mathrm{ng}$ and a detection limit of $51.53 \pm 0.43 \mathrm{pg} / \mathrm{mL}$. These results have been validated through in vitro investigations using real saliva samples spiked with IL8.
\end{abstract}

\section{Introduction}

The frantic working schedules, worldwide witnessed by human race, are associated with the increasing incidences of chronic diseases such as diabetes, stress, cancer, and other metabolic disorders. Oral squamous cell carcinoma (OSCC) is highly prevalent globally and is a major cause for high mortality rate $^{[1]}$ with a higher number in south-east Asian countries. ${ }^{[2]}$ It is one of the common forms of head and neck cancer that involves the formation of cancerous lesions around the oral cavity and oropharynx. ${ }^{[3]}$ Major risk factors include tobacco usage, alcohol consumption, smoking, and human papillomavirus (HPV) infection, while other less prominent factors are poor oral hygiene, improper diet, microbial infection, chronic exposure to sun, or certain chemicals. ${ }^{[3]}$ The delay in diagnosis of the onset of disease remains one of the major contributors to the low survival rate due to oral cancer. In addition to this, the classical methods are invasive, labor-intensive, and expensive making the detection process quite cumbersome for routine diagnosis. ${ }^{[4]}$ This poses a strong need to develop easy to use, fast, and accurate diagnostic tools. To this end, the point-of-care devices, such as biosensors, are the preferred options in healthcare services.

With the identification of various oral cancer-specific biomarkers (proteins expressed by cancerous cells that are indicative of its progress), the prospect of early-stage detection and treatment follow-ups may witness a paradigm shift. ${ }^{[5]}$ Electrochemical detection of tumor-specific biomarkers through specific biosensing devices promises for point-of-care and early detection of oral cancer. ${ }^{[6]}$ Interleukin-8 (IL8) is one such biomarker from the family of cytokines that plays a vital role in tumor growth and metastatic processes in many cancer types. ${ }^{[7]}$ IL8 marks the oral cancer progression with its significantly high levels of expression in saliva $(720 \mathrm{pg} / \mathrm{mL})$ of OSCC patients compared to that in healthy persons $(250 \mathrm{pg} / \mathrm{mL}){ }^{[8]}$ This wide difference in the expression level of IL8 in saliva makes it an intriguing tool for developing noninvasive biosensor for salivary OSCC detection. This diagnostic tool may promise for accurate and reliable oral cancer diagnosis as well as its monitoring throughout the treatment procedures.

The amalgamation of nanomaterials-based transducer systems into electrochemical biosensing devices has recently attracted a lot of attention of the researchers due to their ability to provide fast, simple, and accurate measurement of biomarkers present at very low concentrations in the body fluids. ${ }^{[9,10]}$ Among various nanomaterials proposed, graphene oxide (GO), a planar lattice structure comprising of $\mathrm{sp}^{2}$-bonded carbon in a honeycomb network along with few $\mathrm{sp}^{3}$-bonded carbon attached to functional groups, exhibits outstanding properties, namely high surface area, mechanical strength, conductivity, and charge carrier mobility, making it a preferred nanomaterial for biosensing applications. ${ }^{[1]}$ Moreover, new prototypes involving conjugation of nanoparticles with enhanced heterogeneous electron-transport properties on GO sheets (or its derivatives) are being fervently looked upon to attain advancement in biosensing capability in terms of specificity and disease monitoring time. ${ }^{[12,13]} \mathrm{ZnO}$ nanoparticles are direct bandgap semiconductors known for high charge transfer capabilities and biocompatible nature with high surface area to volume ratio that provide a suitable microenvironment for immobilization, as well as stability of biomolecules on their surface. ${ }^{[14]}$ It was envisioned that integration of $\mathrm{GO}$ (or its derivatives) with $\mathrm{ZnO}$ nanoparticles 
could significantly raise the sensitivity of the electrochemical biosensors.

Here, we report the synthesis of zinc oxide-reduced graphene oxide $(\mathrm{ZnO}-\mathrm{rGO})$ nanocomposite and its application as transducer matrix for the fabrication of a label-free, noninvasive, and cost-effective immunosensing platform for sensitive detection of oral cancer biomarker (IL8). The immunosensor showed successful detection of oral cancer biomarker "IL8" at sub-picomolar ranges with very high sensitivity, selectivity, and good stability. The enhanced electron-transfer property exhibited by $\mathrm{ZnO}-\mathrm{rGO}$ nanocomposite allowed efficient detection of IL8 in saliva as well. The immunosensor represents an edge over classical diagnosis methods for faster detection and clinical evaluation of tumor progression in OSCC patients.

\section{Materials and methods}

\section{Reagents and techniques}

The chemicals used for the synthesis of GO, i.e., potassium permanganate, sulfuric acid, sodium nitrate, hydrogen peroxide, were obtained from Merck India Pvt. Ltd., while graphite powder was provided by Acros Organics. $\mathrm{ZnO}$ nanoparticles were prepared using zinc acetate dihydrate and lithium hydroxide as precursors obtained from Sigma-Aldrich India. A 1:1 (v/v) composition of $N$-ethyl- $N^{\prime}$-(3-dimethylaminopropyl) carbodiimide hydrochloride (EDC) and $N$-hydroxysuccinimide (NHS) obtained from Sigma-Aldrich India was used for activation of carboxylic groups in $\mathrm{ZnO}-\mathrm{rGO}$ composite. Other chemicals used for preparation of Zobell's solution (potassium hexacyanoferrate(II) trihydrate, potassium hexacyanoferrate(III), potassium chloride), phosphate buffer saline (PBS; potassium phosphate monobasic, sodium phosphate dibasic dihydrate, sodium chloride), and blocking agent (ethanolamine) were of ACS grade, also purchased from Sigma-Aldrich. Indiumcoated tin oxide (ITO) substrates were provided by Macwin, India. The target antigen IL8 and its corresponding antibody, i.e., polyclonal IL8 antibody derived from rabbit, were purchased from Bioss, USA.

The physicochemical characterizations were performed using powder $\mathrm{x}$-ray diffractometry (XRD) [model used: Rigaku miniflex 300 containing $\mathrm{Cu} \mathrm{K}_{\alpha}$ x-ray source], UVVisible (UV-Vis) spectrophotometry [model used: Agilent Cary 5000], energy-dispersive x-ray spectroscopy (EDX) [model used: Zeiss EVOMA10], and transmission electron microscopy (TEM) [model used: Tecnai G2 F30 STWIN]. Cyclic voltammetry $(\mathrm{CV})$ and differential pulse voltammetry (DPV) techniques were used to perform electrochemical measurements on PalmSens3 potentiostat in conjugation with a three-electrode system where silver/silver chloride $(\mathrm{Ag} / \mathrm{AgCl})$ electrode and Pt-mesh wire, respectively, were used as reference and counter electrodes, while fabricated nanocompositebased electrodes were applied as working electrodes.

\section{Synthesis of ZnO-rGO nanocomposite}

The $\mathrm{ZnO}-\mathrm{rGO}$ nanocomposite was synthesized from $\mathrm{GO}$ and $\mathrm{ZnO}$ nanoparticles using the wet chemical route. Firstly, modified Hummer's method reported by Cote et al. ${ }^{[15]}$ was applied for the synthesis of highly exfoliated two-dimensional powdered GO. The powdered GO was uniformly dispersed into ethanol through sonication to prepare $1 \mathrm{mg} / \mathrm{mL}$ GO solution. Then, $\mathrm{ZnO}$ nanoparticles of $\sim 3-5 \mathrm{~nm}$ were synthesized using the sol-gel process reported elsewhere ${ }^{[16]}$ with $0.13 \mathrm{M}$ zinc acetate dihydrate (as $\mathrm{Zn}^{2+}$ ion source) and $0.34 \mathrm{M}$ lithium hydroxide monohydrate (as $\mathrm{OH}^{-}$ions source) solutions prepared in ethanol. Finally, $3 \mathrm{~mL}$ of as-synthesized ethanolic dispersion of $\mathrm{ZnO}$ nanoparticles was mixed with $50 \mathrm{~mL}$ of $1 \mathrm{mg} / \mathrm{mL}$ ethanolic solution of GO and stirred overnight at room temperature. The overnight reaction led to the formation of $\mathrm{ZnO}$ nanoparticles on defect sites of $\mathrm{rGO}$ that was formed during the reaction from $\mathrm{GO}$ due to reducing properties of lithium hydroxide monohydrate present in the reaction mixture. The $\mathrm{ZnO}-\mathrm{rGO}$ nanocomposite solution so obtained was utilized further for bioelectrode preparation.

\section{Fabrication of bioelectrodes and response measurements}

The thin films of $\mathrm{ZnO}-\mathrm{rGO}$ nanocomposite were deposited onto ITO-coated glass substrates $\left(6 \times 15 \mathrm{~mm}^{2}\right)$ using the spin coating technique. Then, $50 \mu \mathrm{L}$ of $1: 1 \mathrm{v} / \mathrm{v}$ EDC $(0.5 \mathrm{M})-\mathrm{NHS}$ $(0.1 \mathrm{M})$ mixture was dropcasted on the film surface and left undisturbed for $30 \mathrm{~min}$. The electrodes were washed with $50 \mathrm{mM}$ PBS and then treated with $20 \mu \mathrm{L}$ of $1 \mu \mathrm{g} / \mathrm{mL}$ of IL8 antibodies followed by incubation for $4 \mathrm{~h}$. After the immobilization of antibodies, the films were again washed with PBS and then coated with $1 \mathrm{M}$ ethanolamine to block the unbound active sites on $\mathrm{ZnO}-\mathrm{rGO}$ surface. The electrochemical activity of the films before and after surface modification was determined using the $\mathrm{CV}$ technique. The prepared bioelectrodes (AntiIL8/ZnO-rGO/ITO) were finally exposed to various concentrations of IL8 antigen for 10 min duration for which the response studies were assessed using the DPV method. The overall fabrication process of Anti-IL8/ZnO-rGO/ITO immunoelectrodes and the electrochemical setup used for conducting the electrochemical studies are demonstrated with the aid of a schematic shown in Fig. 1.

\section{Results and discussion}

\section{Characterization of ZnO-rGO nanocomposite}

The structural and morphological features of the synthesized $\mathrm{ZnO}-\mathrm{rGO}$ nanocomposite were investigated using various characterization techniques, i.e., Powder XRD, UV-Vis spectroscopy, EDX and TEM. X-ray diffraction pattern [Fig. 2(a)] of the $\mathrm{ZnO}-\mathrm{rGO}$ nanocomposite was measured in order to explore its crystal structure. The XRD peaks in the $2 \theta$ range $30^{\circ}-80^{\circ}$ exhibited peaks related to (100), (002), (101), (102), (110), (103), (112), (202) planes that matched well with the hexagonal wurtzite structure of $\mathrm{ZnO}$ nanoparticles indexed with JCPDS card no. 36-1451. ${ }^{[17]}$ A broad hump at $2 \theta \sim 23.8^{\circ}$ was also observed that indicated the formation of rGO in the nanocomposite system. ${ }^{[18]}$ 


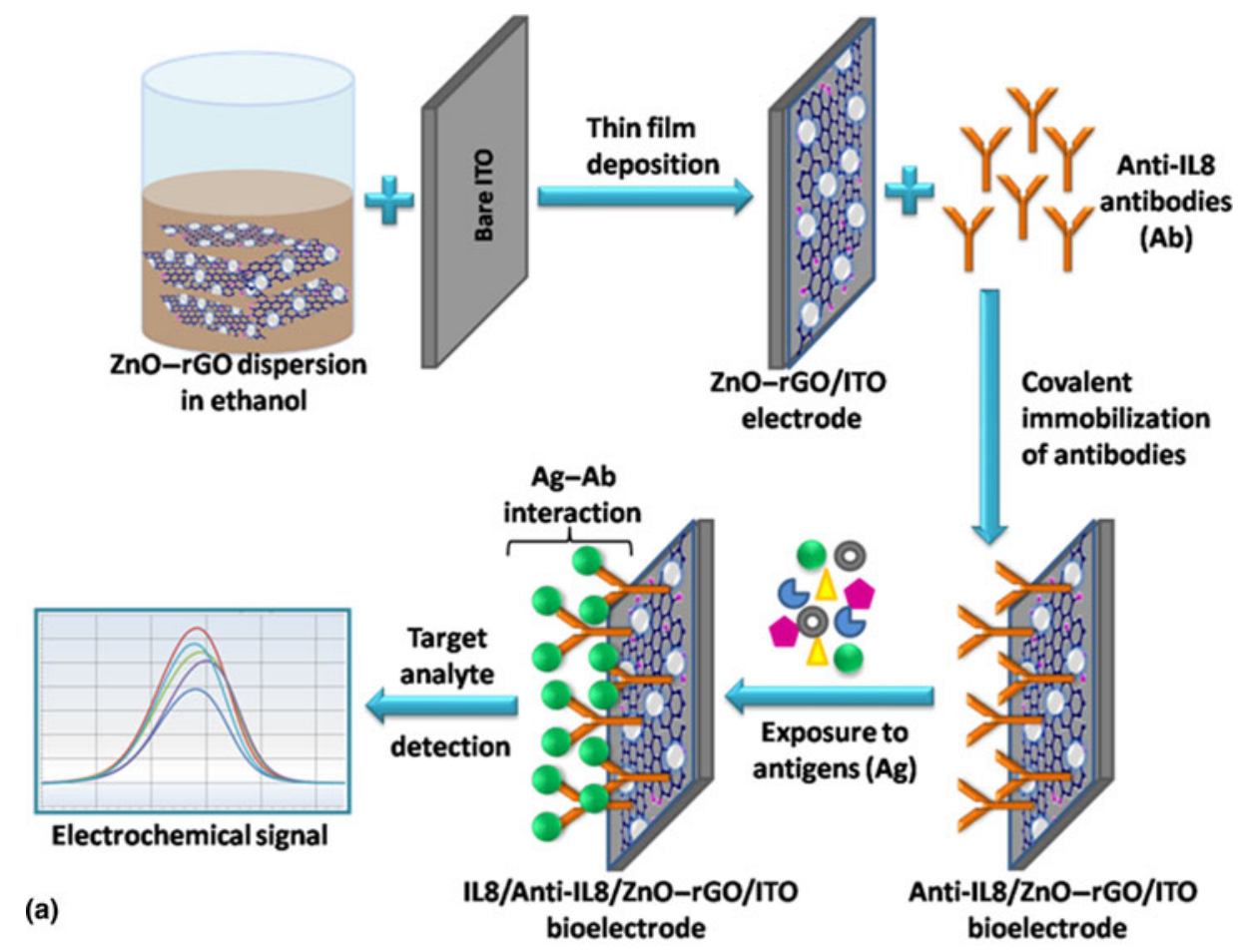

(a)

Electrochemical signal

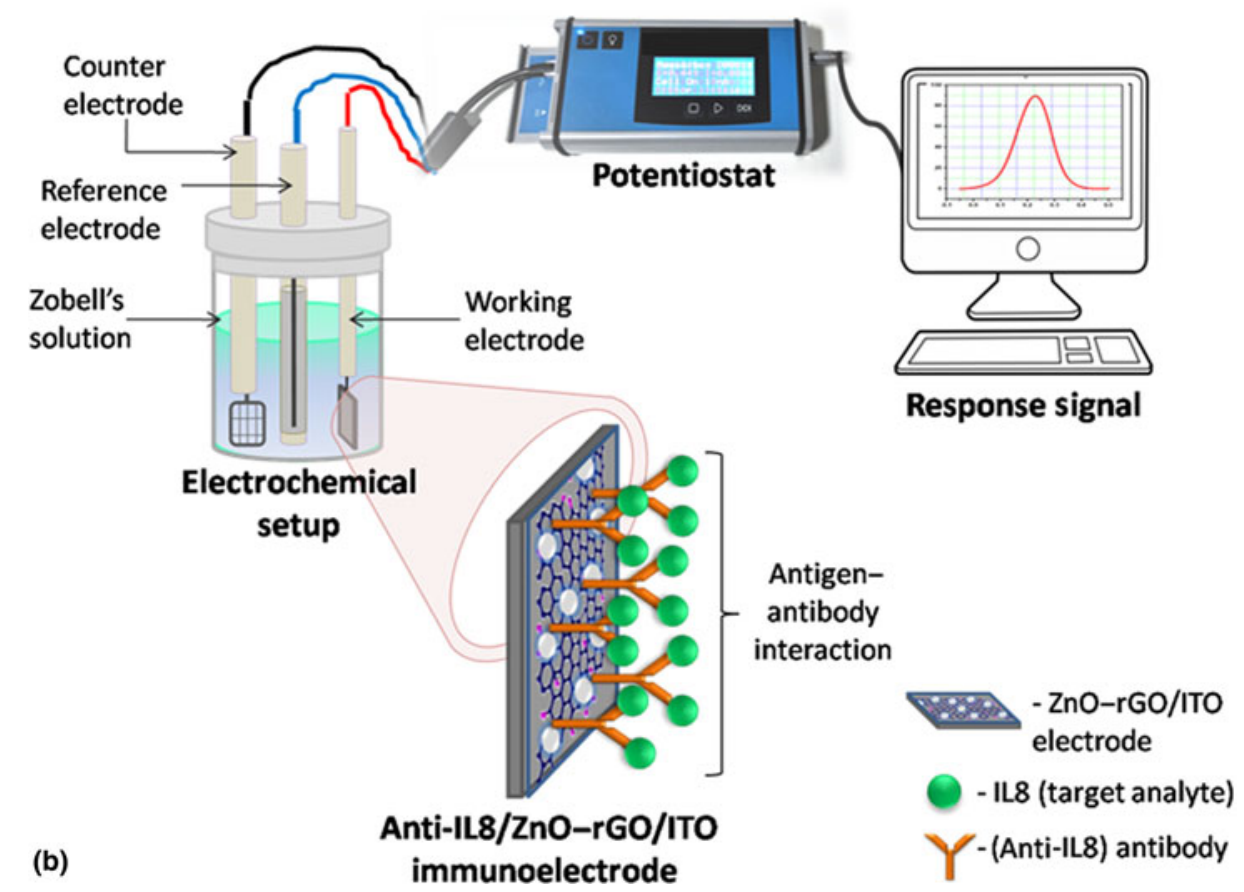

Figure 1. Schematic diagram showing (a) the fabrication process of Anti-IL8/ZnO-rGO/ITO immunosensor and (b) the electrochemical setup applied for recording the electro-analytical measurements of the prepared immunosensor.

Figure 2(b) shows the UV-Vis spectrum of the $\mathrm{ZnO}-\mathrm{rGO}$ nanocomposite that comprised of red-shifted absorption peak related to $n-\pi^{*}(256 \mathrm{~nm})$ transition occurring in $\mathrm{rGO}$ along with the characteristic absorption peak of $\mathrm{ZnO}$ nanoparticles at $347 \mathrm{~nm}$ indicating the conjugation of $\mathrm{ZnO}$ nanoparticles with GO. ${ }^{[19]}$ The optical bandgap of $\mathrm{ZnO}-\mathrm{rGO}$ nanocomposite was determined from the extrapolation of linear portion to the intercept of $x$-axis in a plot between $(\alpha h v)^{2}$ versus energy $(\mathrm{eV})$, i.e., Tauc's plot. ${ }^{[20]}$ The prepared nanocomposite showed a smaller bandgap value (i.e., $3.36 \mathrm{eV}$ ) in comparison to 


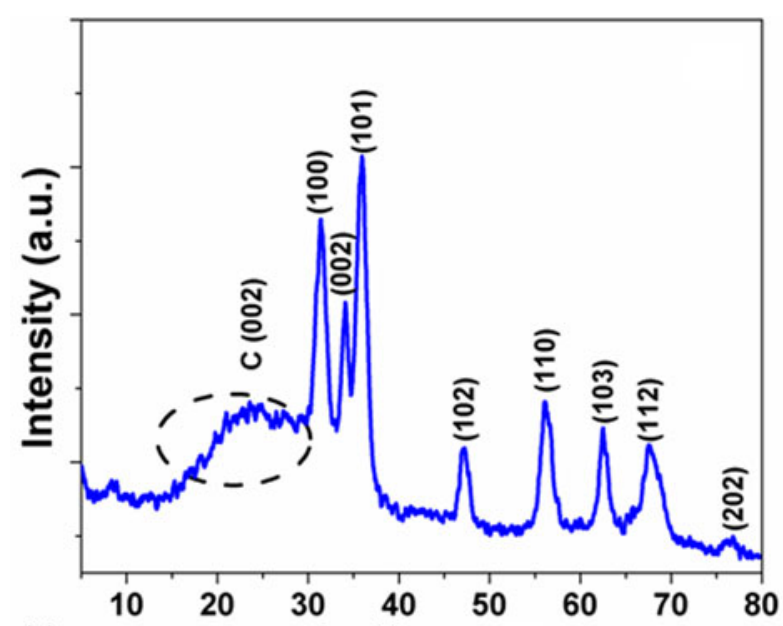

(a)
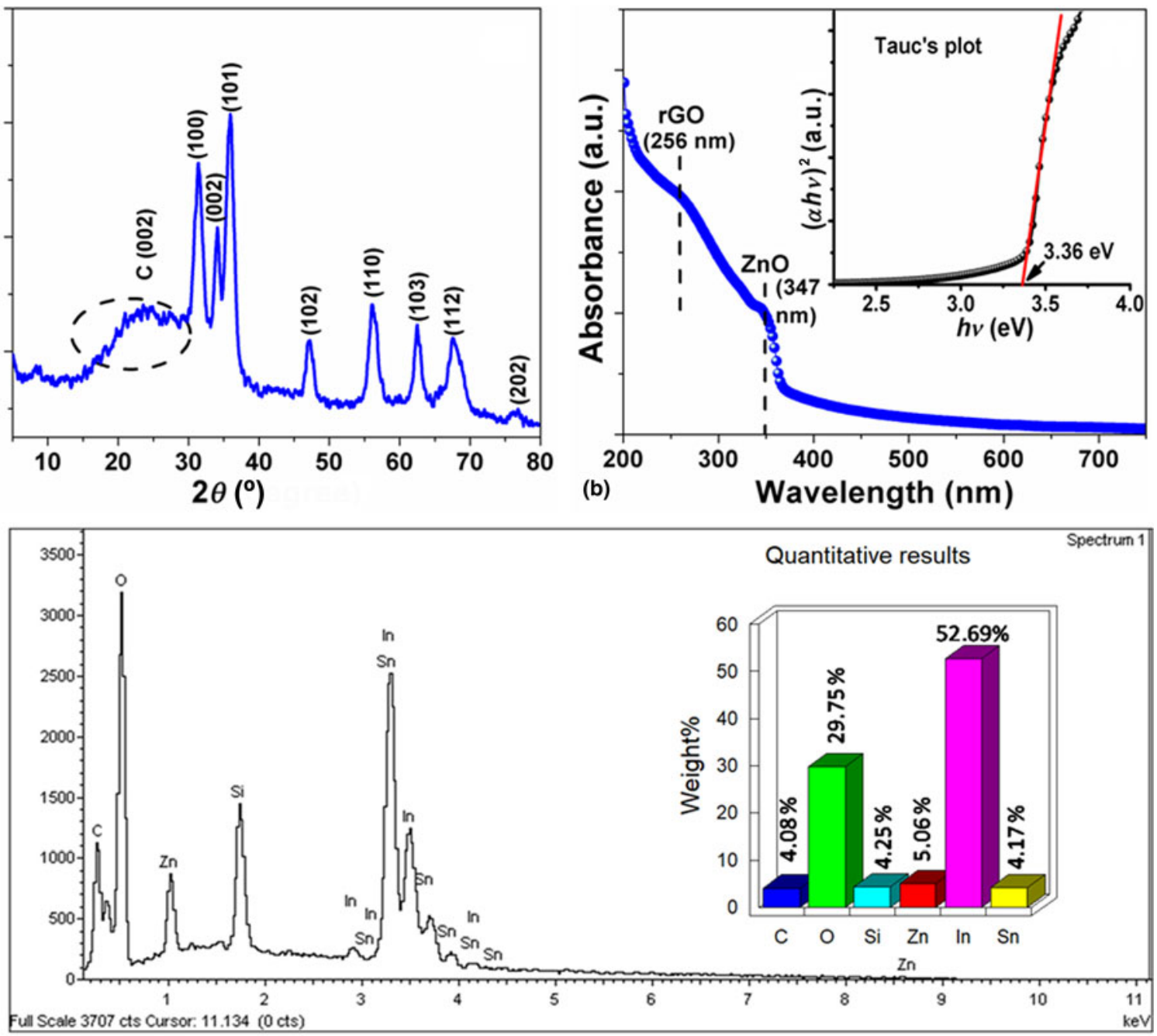

(c) Eull Scale 3707 cts Cursor. 11.134 (0 cts)
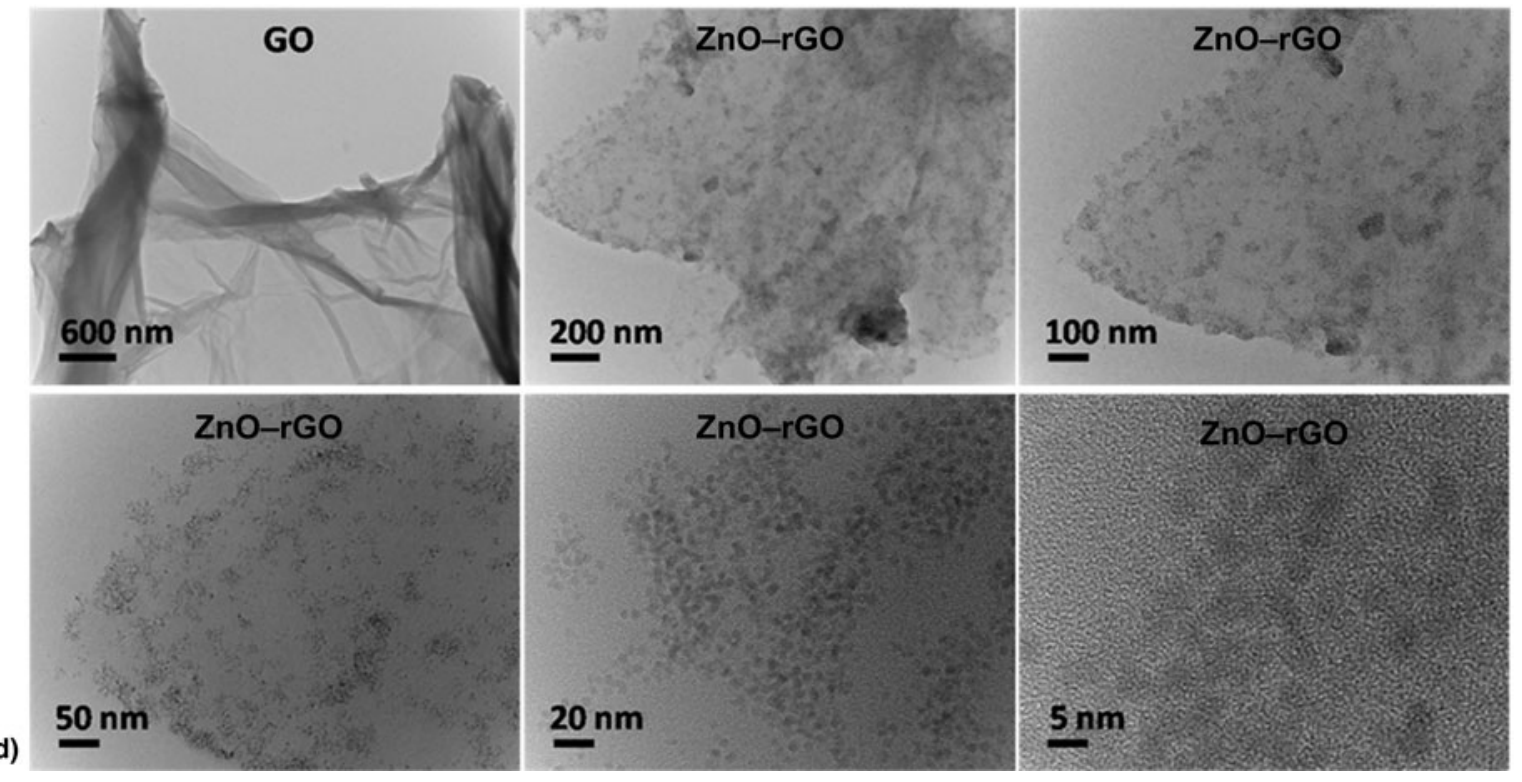

Figure 2. (a) Powder XRD pattern and (b) solution-based UV-Vis spectrum of ZnO-rGO nanocomposite. Inset shows Tauc's plot associated with the obtained optical behavior of ZnO-rGO nanocomposite. (c) EDX spectra and bar graph showing the chemical composition of Zn0-rGO nanocomposite film on ITO. (d) TEM images of GO and ZnO-rGO nanocomposite solutions deposited on 200-mesh copper grids. 
unbound $\mathrm{ZnO}$ nanoparticles (bandgap- $3.62 \mathrm{eV}$ ) (figure not shown) indicating toward facile charge transfer capability of the composite.

The elemental composition of the prepared nanocomposite was identified by subjecting the thin films of $\mathrm{ZnO}-\mathrm{rGO}$ on ITO-coated glass (prepared using the spin-coating technique) to EDX analysis. The EDX spectrum [Fig. 2(c)] revealed peaks related to $\mathrm{C}$ and $\mathrm{Zn}$ belonging to the prepared nanocomposite, while the other peaks denoting In, Sn and Si were coming from the ITO substrate. The high intensity peak for $\mathrm{O}$ element was obtained due to contribution from both the nanocomposite and the ITO substrate. The quantitative distribution of these elements is depicted through the bar graph in Fig. 2(c).

The morphology of the $\mathrm{ZnO}-\mathrm{rGO}$ nanocomposite was viewed at an atomic scale using a panel of TEM images recorded at different scales [Fig. 2(d)]. The TEM image of GO showed crumpled sheet-like structure with high transparency, while the TEM image of ZnO-rGO showed the dense distribution of monodispersed $\mathrm{ZnO}$ nanoparticles $(\sim 3-5 \mathrm{~nm}$ in size) onto the sheets of rGO further confirming the selfassembly of $\mathrm{ZnO}$ onto $\mathrm{rGO}$ surface.

\section{Electrochemical studies}

The electrochemical activity of the prepared thin films was evaluated by employing the $\mathrm{CV}$ technique for studying the redox reaction of ferro-ferri couple (i.e., Zobell's solution) at varying scan rates $(0.02-0.24 \mathrm{~V} / \mathrm{s})$ using $\mathrm{ZnO}-\mathrm{rGO} / \mathrm{ITO}$ films as working electrodes [Fig. 3]. The recorded CV curves showed an increase in redox current with increasing scan rates in accordance with the Randles-Sevcik equation [Eq. (1)] that defines a direct relationship between current and square roots of scan rates:

$$
I_{\mathrm{p}}=\left(2.69 \times 10^{5}\right) n^{3 / 2} A C D^{1 / 2} v^{1 / 2}
$$

where $I_{\mathrm{p}}$ is the anodic/cathodic peak current, $n$ is the number of electrons involved in the redox process, $A$ is the active surface area of electrode, $C$ is the molar concentration of the redox species, $D$ is the diffusion coefficient specific to the redox process, and $v$ is the applied scan rate value.

The films revealed quasi-reversible electrochemical characteristics as evident from the shift in potential values, $E_{\mathrm{p}}$ (a positive shift in oxidation cycle and a negative shift in reduction cycle), corresponding to anodic $\left(I_{\mathrm{pa}}\right)$ and cathodic $\left(I_{\mathrm{pc}}\right)$ peak currents with a successive increase in scan rate. The ratio for forward-to-reverse peak currents lying in the range $1<I_{\mathrm{pa}} / I_{\mathrm{pc}}$ $<1.29$ additionally verified the occurrence of quasi-reversible electrochemistry at $\mathrm{ZnO}-\mathrm{rGO} / \mathrm{ITO}$ electrode where the rates of electron transfer and mass transfer are comparable. ${ }^{[21]}$ Further, the graph given in Fig. 3 (inset) illustrates the straight line behavior of peak currents $(\mu \mathrm{A})$ with respect to square root of scan rates $\left(\mathrm{V}^{1 / 2} / \mathrm{s}^{1 / 2}\right)$ having correlation coefficient, $R^{2}=0.9998$ and $0.9996(<1)$, suggesting the diffusion-based electron-transport process in the vicinity of the electrode. ${ }^{[21,22]}$

The $\mathrm{ZnO}-\mathrm{rGO} / \mathrm{ITO}$ electrodes were subjected to controlled immobilization process for covalent attachment of Anti-IL8

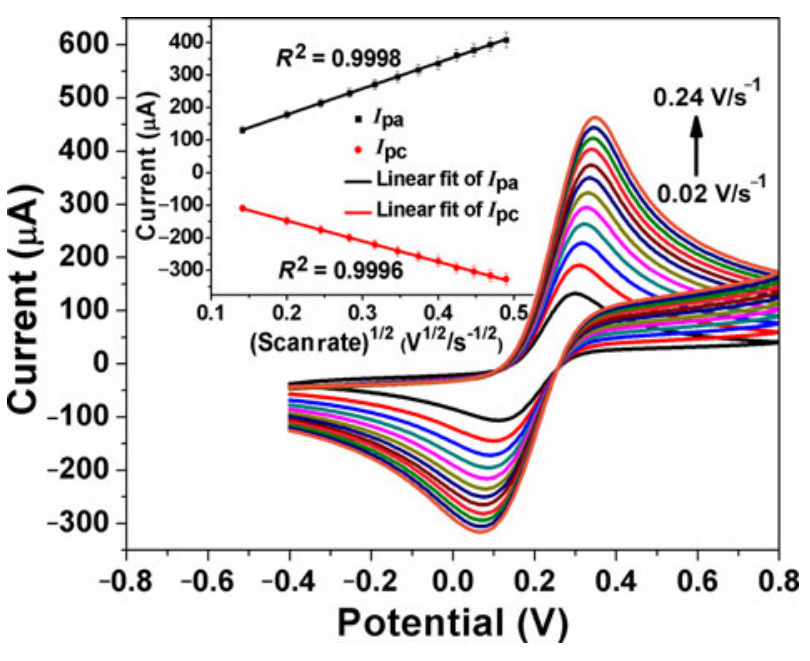

Figure 3. CV curves recorded at ZnO-rGO/ITO electrode for $3 \mathrm{mM}$ Zobell's solution with varying scan rates. Inset shows the plot of anodic/cathodic peak currents versus square root of scan rates.

antibodies on their surface. The successful immobilization of the antibodies on $\mathrm{ZnO}-\mathrm{rGO} / \mathrm{ITO}$ electrodes was determined through FTIR spectroscopic measurements, given in Supplementary Fig. S1(a), that showed peaks related to stretching frequencies of amide $\mathrm{C}=\mathrm{O}$ and $\mathrm{N}-\mathrm{H}$ bonds, thus confirming the formation of amide linkage between antibody and the nanocomposite system. The resulting immunoelectrodes, Anti-IL8/ ZnO-rGO/ITO, were tested for their response toward IL8 at a concentration range of $100 \mathrm{fg} / \mathrm{mL}-5 \mathrm{ng} / \mathrm{mL}$ using the DPV technique with applied pulse potential $0.02 \mathrm{~V}$ and $0.07 \mathrm{~s}$ of pulse time [Fig. 4(a)]. DPV curves, recorded to account for the antibody-antigen binding event, showed sharp well-defined oxidation peaks whose current $\left(I_{\mathrm{p}}\right)$ values decreased with increasing analyte concentrations due to the presence of low conducting protein layer at the electrode interface. The optimal response time of the immunoelectrode toward IL8 was found to be $10 \mathrm{~min}$. A calibration curve between peak current and analyte concentration was constructed [Fig. 4(a) inset], giving a linear response of current signal with the exposed IL8 concentration with the linear regression equation:

$$
I_{\mathrm{p}}=-0.01246[\mathrm{IL} 8 \text { conc. }]+62.6864, \quad R^{2}=0.99303
$$

The sensitivity of Anti-IL8/ZnO-rGO/ITO immunosensor as derived from the slope of the calibration curve was found to be $12.46 \pm 0.82 \mu \mathrm{A} \mathrm{mL} / \mathrm{ng}$. The value for the limit of detection (LOD) of the immunosensor was calculated to be $51.53 \pm 0.43 \mathrm{pg} / \mathrm{mL}(n=3)$ using the following equation ${ }^{[23,24]}$ :

$$
\mathrm{LOD}=k \times \frac{\sigma}{S}
$$

where $k=3$ corresponding to the confidence level parameter, $\sigma$ is the standard deviation value for response signals obtained 

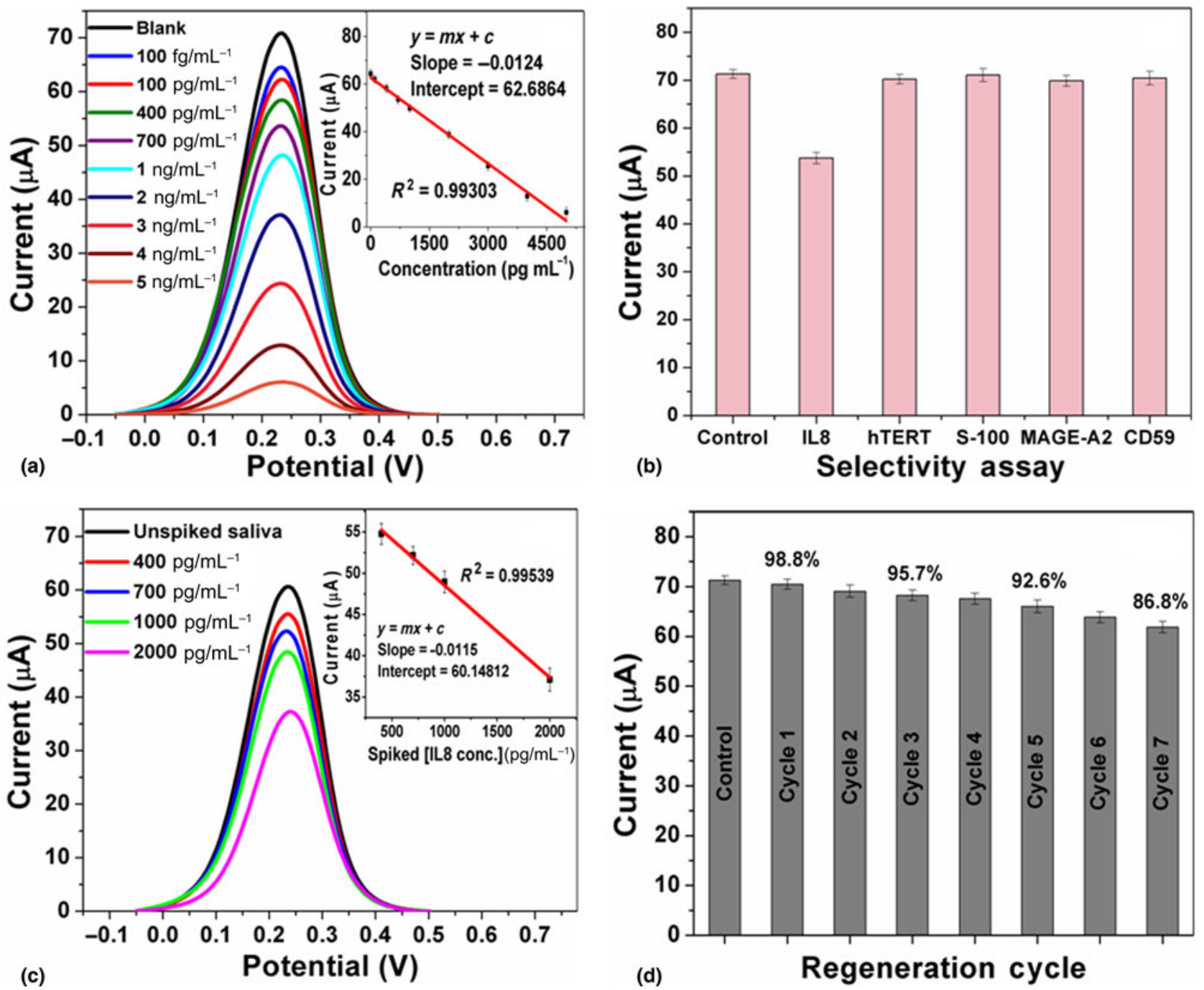

Figure 4. (a) DPV curves showing the response of Anti-IL8/Zn0-rGO/ITO immunoelectrode toward different concentrations of standard IL8 antigen (prepared in $50 \mathrm{mM}$ PBS) in $3 \mathrm{mM}$ Zobell's solution. Inset shows the corresponding calibration curve between the response current and concentration. (b) Bar graph showing the immunosensor response toward nontarget analytes in comparison with response toward IL8. (c) DPV curves showing the performance of immunosensor toward spiked IL8 concentrations in saliva. Inset shows the calibration plot of current versus concentration obtained for spiked saliva samples. (d) Bar graph illustrating the activity of the immunosensor after seven regeneration cycles.

in the absence of an analyte, and $S$ is the slope of the calibration plot which is equal to the sensitivity of the electrode.

Specificity is an important parameter that the immunosensor must exhibit in order to prevent false positives. To ensure the specificity of our fabricated immunosensor, it was exposed to other nontarget biomarker proteins, i.e., hTERT, S-100, MAGE-A2, and CD59, each having concentration of $700 \mathrm{pg} / \mathrm{mL}$ [Fig. 4(b)]. It was found that the oxidation peak current value for each of the nonspecific analytes was comparable to the response signal of the blank sample (i.e., control sample not containing any analyte) indicating negligible interference of the nonspecific analytes on the response of the immunosensor due to their noninteraction with the immobilized antibody on the electrode surface. On the other hand, the current decreased significantly when the immunosensor was exposed to the target analyte, i.e., IL8, confirming high specificity of immunosensor toward IL8 antigen. The high sensitivity and specificity exhibited by the immunosensor validates its superior electrochemical performance attributable to the structural defects induced low energy gap in the nanocomposite causing fast electron mobility.

In order to establish the diagnostic capability of the fabricated immunosensor, its performance was investigated in real biological samples. Four different concentrations $(400 \mathrm{pg} / \mathrm{mL}$, $700 \mathrm{pg} / \mathrm{mL}, 1 \mathrm{ng} / \mathrm{mL}$, and $2 \mathrm{ng} / \mathrm{mL}$ ) of standard IL8 were spiked into the saliva samples (in its undiluted form) collected from healthy volunteers and exposed to the immunosensor for 10 min duration. The response of the immunosensor recorded 
using a DPV technique [Fig. 4(c)] revealed unhindered detection of the spiked IL8 concentrations with a calibration curve fitting in the given linear regression equation:

$$
I_{\mathrm{p}}=-0.01157[\mathrm{IL} 8 \text { conc. }]+60.14812, R^{2}=0.99539
$$

The sensitivity $(11.57 \pm 0.72 \mu \mathrm{A} \mathrm{mL} / \mathrm{ng})$ obtained from the calibration curve [Fig. 4(c) inset] in case of saliva samples was quite comparable to that obtained for standard IL8 samples, thus proving the negligible matrix effect on the functioning of the immunoelectrodes. Further, the immunosensor exhibited appreciable recovery values in the range $95 \%-$ $100 \%$, for all the spiked IL8 concentrations with relative standard deviation (RSD) $<4 \%$, signifying its competency for diagnosis of biological samples.

The regeneration capability of the immunosensor was also determined by subjecting the immunoelectrodes to $30 \mathrm{mM}$ $\mathrm{NaOH}$ solution ( $\mathrm{pH}$ 12) for $90 \mathrm{~s}$ to allow breaking of antigen-antibody interaction and then recording the oxidation cycle $(n=3)$ using the DPV. As evident from the bar graph [Fig. 4(d)] showing the current obtained after each regeneration cycle, the immunosensor retained $98.8 \%$ activity after the first regeneration cycle. The activity of the immunosensor then decreased to $95.7 \%$ after the third cycle and further down to $92.6 \%$ in the fifth cycle. After five regeneration cycles, the biochemical activity reduced significantly, implying that the immunosensor could effectively work for five successive uses.

The stability of the immunosensor was also analyzed by recording its response in triplicate toward $700 \mathrm{pg} / \mathrm{mL}$ of IL8 after every 10 days. The obtained results testified reliable performance of the immunosensor for up to 70 days when stored at $4{ }^{\circ} \mathrm{C}$ temperature. Repeatability of results is crucially important for the development of a biosensor that could be applied for reliable clinical diagnosis. To evaluate the repeatability of our fabricated immunosensor Anti-IL8/ZnO-rGO/ITO, we have presented the DPV results of 15 sets of measurements for four different concentrations of IL8 $(400 \mathrm{pg} / \mathrm{mL}, 700 \mathrm{pg} / \mathrm{mL}$, $1 \mathrm{ng} / \mathrm{mL}$, and $2 \mathrm{ng} / \mathrm{mL}$ ) in Supplementary Fig. S3. The overall RSD below $3.2 \%$ for all the concentrations of IL8 advocated the high rates of repeatability in the results. However, the little uncertainty recorded in the current values (given in Supplementary Table S1) for each of the exposed IL8 concentrations possibly arises from the non-ambient experimental conditions, open-ended materials processing practices and handling errors during antibody fabrication steps.

\section{Conclusion and future outlook}

We have highlighted the successful preparation of a robust immunosensing platform, Anti-IL8/ZnO-rGO/ITO, and its efficiency to detect oral cancer-specific biomarker IL8 in saliva samples. The atomically thin rGO sheets provided a high surface area and an ample amount of carboxylic groups that enabled the covalent attachment of antibodies on its surface. The overall smaller band structure and electronic synergy of rGO and $\mathrm{ZnO}$ in the nanocomposite matrix led to the achievement of high sensitivity $(12.46 \pm 0.82 \mu \mathrm{A} \mathrm{mL} / \mathrm{ng})$ and a $\operatorname{LOD}$ value $(51.53 \pm 0.43 \mathrm{pg} / \mathrm{mL})$ which is 15 times lower than the physiochemical level of IL8 in saliva of oral cancer patients. Additionally, the fabricated immunosensor showed a remarkable response toward IL8 in saliva samples. The results obtained from the designed biosensor are reproducible and serve as a prototype for designing of noninvasive biosensing platform for diagnosis of other cancerous biomarkers through a single- or multianalyte-based detection mode.

\section{Supplementary material}

The supplementary material for this article can be found at https://doi.org/10.1557/mrc.2019.138.

\section{Acknowledgments}

The authors duly acknowledge support from the Director, CSIR-NPL and CSIR-Network project BSC0112. S.V. is grateful for the funding provided by CSIR (31/01/(0510)/2018EMR-1) India.

\section{References}

1. R.L. Siegel, K.D. Miller, and A. Jemal: Cancer statistics, 2018. CA Cancer J. Clin. 68, 7-30 (2018).

2. S.V. Krishna Rao, G. Mejia, K. Roberts-Thomson, and R. Logan: Epidemiology of oral cancer in Asia in the past decade - an update (2000-2012). Asian. Pac. J. Cancer Prev. 14, 5567-5577 (2013).

3. C. Rivera: Essentials of oral cancer. Int. J. Clin. Exp. Pathol. 8, 1188411894 (2015).

4. R. Mehrotra and D.K. Gupta: Exciting new advances in oral cancer diagnosis: avenues to early detection. Head Neck Oncol. 3, 33 (2011).

5. S.B. Nimse, M.D. Sonawane, K.-S. Song, and T. Kim: Biomarker detection technologies and future directions. Analyst 141, 740-755 (2016).

6. B.V. Chikkaveeraiah, A.A. Bhirde, N.Y. Morgan, H.S. Eden, and X. Chen: Electrochemical immunosensors for detection of cancer protein biomarkers. ACS Nano 6, 6546-6561 (2012).

7. H. Watanabe, M. Iwase, M. Ohashi, and M. Nagumo: Role of interleukin-8 secreted from human oral squamous cell carcinoma cell lines. Oral Oncol. 38, 670-679 (2002).

8. M.A. St. John, Y. Li, X. Zhou, P. Denny, C.M. Ho, C.D. Montemagno, W. Shi, F. Qi, B. Wu, U. Shinha, R. Jordan, L. Wolinsky, N.H. Park, H. Liu, E. Abemayor, and D.T.W. Wong: Interleukin 6 and interleukin 8 as potential biomarkers for oral cavity and oropharyngeal squamous cell carcinoma. Arch. Otolaryngol. Head Neck Surg. 130, 929-935 (2004).

9. U. Anik and S. Timur: Towards the electrochemical diagnosis of cancer: nanomaterial-based immunosensors and cytosensors. RSC Adv. 6, 111831-111841 (2016).

10.J.F. Rusling, G. Sotzing, and F. Papadimitrakopoulos: Designing nanomaterials-enhanced electrochemical immunosensors for cancer biomarker proteins. Bioelectrochem. 76, 189-194 (2009).

11. L. Wang, Q. Xiong, F. Xiao, and H. Duan: 2D nanomaterials based electrochemical biosensors of cancer diagnosis. Biosens. Bioelectron. 89, 136151 (2017)

12. S. Verma, A. Singh, A. Shukla, J. Kaswan, K. Arora, J. Ramirez-Vick, P. Singh, and S.P. Singh: Anti-IL8/AuNPs-rGO/ITO as an immunosensing platform for noninvasive electrochemical detection of oral cancer. ACS Appl. Mater. Interfaces 9, 27462-27474 (2017).

13. N. Pachauri, K. Dave, A. Dinda, and P.R. Solanki: Cubic $\mathrm{CeO}_{2}$ implanted reduced graphene oxide-based highly sensitive biosensor for noninvasive oral cancer biomarker detection. J. Mater. Chem. B 6, 30003012 (2018)

14. S.K. Arya, S. Saha, J.E. Ramirez-Vick, V. Gupta, S. Bhansali, and S.P. Singh: Recent advances in $\mathrm{ZnO}$ nanostructures and thin films for biosensor applications: review. Anal. Chim. Acta 737, 1-21 (2012). 
15. L.J. Cote, F. Kim, and J. Huang: Langmuir-Blodgett assembly of graphite oxide single layers. J. Am. Chem. Soc. 131, 1043-1049 (2009).

16. L. Spanhel and M.A. Anderson: Semiconductor clusters in the sol-gel process: quantized aggregation, gelation, and crystal growth in concentrated Zn0 colloids. J. Am. Chem. Soc. 113, 2826-2833 (1991).

17. Y.-L. Chen, Z.-A. Hu, Y.-Q. Chang, H.-W. Wang, Z.-Y. Zhang, Y.-Y. Yang, and H.-Y. Wu: Zinc oxide/reduced graphene oxide composites and electrochemical capacitance enhanced by homogeneous incorporation of reduced graphene oxide sheets in zinc oxide matrix. J. Phys. Chem. C 115, 2563-2571 (2011).

18. H.N. Tien, V.H. Luan, L.T. Hoa, N.T. Khoa, S.H. Hahn, J.S. Chung, E.W. Shin, and S.H. Hur: One pot-synthesis of reduced graphene oxide-zinc oxide sphere composites and its use as a visible light photocatalyst. Chem. Eng. J. 229, 126-133 (2013).

19. J. Wang, T. Tsuzuki, B. Tang, X. Hou, L. Sun, and X. Wang: Reduced graphene oxide/zinc oxide composite: reusable adsorbent for pollutant management. ACS Appl. Mater. Interfaces 4, 3084-3090 (2012).

20. M.K. Debanath and S. Karmarkar: Study of blueshift of optical band gap in zinc oxide $(\mathrm{ZnO})$ nanoparticles prepared by low-temperature wet chemical method. Mater. Lett. 15, 116-117 (2013).

21. J. Wang: Analytical Electrochemistry, Vol. 272, 3rd ed. (John Wiley and Sons, New Jersey, 2006).

22. A.J. Bard and L.R. Faulkner: Electrochemical Methods Fundamentals and Applications, 2nd ed. (John Wiley and Sons, Inc., USA, 2001).

23. S. Verma, J. Choudhary, K.P. Singh, P. Chandra, and S.P. Singh: Uricase grafted nanoconducting matrix based electrochemical biosensor for ultrafast uric acid detection in human serum samples. Int. J. Biol. Macromol. 130, 333-341 (2019).

24. S. Jain, S. Verma, S.P. Singh, and S.N. Sharma: An electrochemical biosensor based on novel butylamine capped CZTS nanoparticles immobilized by uricase for uric acid detection. Biosens. Bioelectron. 127, 135-141 (2019). 